\title{
Energy-Efficient Cooperative Sensing for Cognitive Wireless Sensor Networks
}

\author{
Hany Ali, Ahmed Khattab, Magdi Fikri \\ Electronics and Electrical Communications Engineering Department \\ Cairo University \\ Giza, Egypt 12613 \\ aalhany@gmail.com, akhattab@ieee.org,magdi.fikri@gmail.com
}

\begin{abstract}
Cognitive radio sensor networks (CRSNs) exploit the cognitive radio concept to allow wireless sensor networks to dynamically access the available channels. However, existing channel sensing techniques developed for cognitive radios are not applicable to the energy-constrained sensor nodes. In this paper, we present two energy-efficient cooperation schemes for CRSNs. The proposed schemes use randomized channel sensing, implicit cooperation, and simplified aggregation to reduce the energy consumed in channel sensing. The proposed implicit OR and implicit AND save up to $55 \%$ of the energy, reduce the decision taking time by $30.6 \%-95 \%$, and achieve similar miss-detection performance compared to their explicit counterparts.
\end{abstract}

Keywords-cognitive radio; wireless sensor networks; energyefficient; cooperative sensing;

\section{INTRODUCTION}

Cognitive radio wireless sensor networks (CRSNs) are one of the candidate areas where cognitive radio $(\mathrm{CR})$ techniques can be used. Research in this area is still in its infancy, but it is progressing rapidly. Adding the CR feature solves the spectrum scarcity problem and benefits from the opportunistic channel usage for bursty traffic. However, the CR concept also brings additional challenges and leads to higher energy consumption, while wireless sensor networks (WSNs) use energy limited devices as it is battery-powered.

The CRSN node differs from CR node from the node, network and protocol architectures points of view. These differences make CR techniques not applicable to CRSNs and need to adapt to the particular CRSN environment. More specifically, channel sensing protocols in CRSNs need to be energy-efficient, scalable, light weight and have minimum channel switching rate. Otherwise, the CRSN will have smaller life time, higher cost and limited number of nodes. Motivated with improving the CRSN energy efficiency, we propose two energy-efficient cooperative sensing techniques. Unlike the recent studies that tackled the energy efficiency of channel sensing in CRSN such as $[1,2]$, we break down and individually address all the sources of energy waste in CRSNs.

In this paper, we present a cross layer design to achieve a green efficient cooperative sensing technique. We redesign the sensing cycle of CR to be compatible with CRSNs, keeping in mind the fact that primary networks (PNs) do not accept interference from the CRSN nodes. This interference comes from the miss detection (MD) of PN transmissions at the CRSN nodes. In order to have a practical design, all factors that affect the sensing cycle - such as channel switching, data transferring, and decision delay - are addressed to match the critical energy requirements of CRSNs. Our results show that using implicit aggregation the energy consumption is reduced to up to $55 \%$ and the decision delay is improved by $30.6 \%$ to $95 \%$ compared to explicit aggregation. The proposed implicit OR (I-OR) rule is more energy-efficient than implicit AND (I-AND) at low PN activity (below 60\%) while I-AND is more efficient at high PN activity. Furthermore, implicit I-AND and I-OR aggregation result in $25 \%$ less and same miss-detection (MD) percentages, respectively, compared to their explicit counterparts. However, the false alarm (FA) percentages in our schemes exceed the explicit rules, yet false alarms do not harm the PNs.

The remainder of the paper is organized as follows. In section II, we present the needed preliminaries and motivation. The proposed implicit cooperative sensing techniques are introduced in Section III. Section IV and V are the performance and the conclusions, respectively.

\section{PRELIMINARIES AND MOTIVATION}

\section{A. System Model}

We consider a distributed wireless CRSN geographically collocated with $N$ primary networks. Each PN operates in a separate channel. Each PN alternates between ON and OFF states. The OFF time represents the absence of PN activity, and can be exploited by the CRSN nodes. The PN activity factor $F_{P}$ is the percentage of time the $\mathrm{PN}$ is in the $\mathrm{ON}$ state relative to the total time, i.e., $F_{P}=T_{O N} /\left(T_{O N}+T_{O F F}\right)$. A CRSN node operates in any of the $N$ channels of the PNs one at a time.

The CRSN is composed of $M$ nodes, referred to as the secondary users (SUs), which are uniformly distributed within the field. We assume high density of the CRSN nodes as the case in typical wireless sensor network applications. All CRSN nodes are considered to be static. Each SU is battery powered, resource constrained and equipped with a single half-duplex transceiver. Deployed sensor nodes are homogenous in terms of hardware and initial battery power. Over time, the sensor nodes may be left out with non-uniform levels of energy.

One common control channel (CCC) exists in the unlicensed band (or using UWB spread spectrum) to be used for coordination between the CRSN nodes. Each of the SU makes its own local decision independently using energy detection [3, 4]. Energy detection is widely adopted in the contexts of cognitive radio and wireless sensor network because it has low complexity and it does not require any apriori knowledge of the primary signals. We consider a synchronous system in which time is divided into frames. A frame $F$ is divided into three periods: sensing $\left(T_{S}\right)$, cooperation $\left(T_{c}\right)$ and transmission $\left(T_{R}\right)$ periods.

\section{B. Explicit Cooperative Sensing}

Existing cooperative sensing [5] requires the cooperating nodes (either the entire node population or a selected subset of 
nodes) to explicitly share all their sensing information in order to make a decision. We refer to such techniques as explicit cooperative sensing. In the sensing phase of a frame, all SU nodes participating in cooperation perform the sensing task for each channel individually as shown Fig. 1 (a). The channel is considered as busy, if the sensed energy level is above a certain threshold. Each node then tunes its radio to the next channel and repeats the process until all channels are sensed.

After sensing the last channel, the SU nodes enter the cooperation phase in which all SUs move to the CCC to send their local decisions to a fusion center (FC) using contention-based channel access as shown in Fig. 2(a). The fusion center is a powerful node with advanced computational capabilities and operates only on the CCC. The FC is responsible for generating a channel map (CM) that uses the gathered information to come up with final decisions regarding the availability of each of the $N$ channels. The $\mathrm{CM}$ is generated by aggregating all SUs data according to a certain strategy. The aggregation strategies are either hard decision strategies or soft decision strategies. In Hard decision strategies, Each SU takes the decision for every channel and convey the decision in one bit. A ' 1 ' means that the PN is ON and a ' 0 ' means the PN is OFF. The FC aggregates these local decisions using logical or statistical rules. There are three widely used aggregation rules: the AND (all the SUs infer the existence of $\mathrm{PN}$ ), OR (at least one SU infers the existence of PN) and majority (MAJ. - at least half the SUs infer the existence of PN) rules.

\section{Motivation}

Explicit sensing techniques are designed for CR networks in which energy consumption is not of a paramount importance. Applying such techniques in CRSNs results in poor performance in terms of the energy consumption, delay, required number of channel switching, and the required computational power [6]. In addition to the scalability problems [7] since a CRSN have a much larger number of nodes than a CR network. In what follows, we briefly explain each of such problems.

1) High Energy Consumption: The CRSN sensing energy is consumed in five activities: (1) sensing the various channels, (2) switching the transceiver hardware to the new frequency channel to be sensed, (3) communicating the sensing results to the FC which energy consumption increases in contention-based transmissions due to collisions and retransmissions, (4) operating in the idle listening mode during the $T_{c}$ period which energy consumption is comparable to the packet reception energy consumption, and (5) receiving the CM message from FC. Reducing the first four energies not only makes CRSN green but also increases the network efficiency, life time, reduces the required resources, and consequently, reduces the cost of the node.

2) Long Decision Making Delay: The PNs activities are evaluated in the $T_{S}$ period and a decision is made and shared at the end of the $T_{c}$ period using the CM. Such a decision is used in the transmission period between the broadcast of a CM and the beginning of the $T_{s}$ period of the next frame. We refer to such interval as the decision validity interval of the $\mathrm{CM}$. If the $\mathrm{PN}$ changes its state during the decision validity interval, then collisions and interference with the PN will occur or available channels will be not utilized by the CRSN. For a more accurate and efficient sensing process, we need to minimize two intervals: (1) the decision making interval which contains the sensing period $T_{S}$ and the cooperation period $T_{C}$, and (2) the decision validity interval of the CM. Reducing the decision validity interval reduces the throughput of the CRSN, and hence, is undesirable.
Consequently, the decision making time should be reduced to better track the activities of the PNs.

3) Aggregation Computational Complexity: Explicit cooperation poses computational resource challenge which is not suitable for CRSN. The aggregation process of the channel sensing information of the several nodes participating in cooperation requires computational resources that scale up with the number of cooperating nodes. This increases the complexity of the FC making it a special node with higher cost. In contrast, a simpler aggregation process will allow any ordinary node (with modest computational capabilities) handles the aggregation process using its resources, and hence, any node could serve as a FC in that case.

4) Scalability Problem: Explicit sensing techniques are not scalable due to two facts. First, sensing a large number of channels requires too long sensing period $T_{S}$. Second, a large number of cooperating SUs requires long contention period $T_{C}$ in order to convey all their information to the FC. The FC waits until all nodes sense all channels then send their information to generate the CM which will be no longer valid after such long delay. Thus, explicit cooperation is not suitable for the dynamic CRSNs. which are typically of high node density unlike traditional CRNs.

This paper aims to design an energy-efficient sensing protocol that is suitable for CRSNs. Our goal is to decrease the energy consumption sources, the decision making interval, the required number of channel switching and the required computational power. We also aim at turning the scalability problem into an advantage by having our protocol benefiting from the large number of nodes as the case with typical CRSN applications. Our approach exploits the correlation between the channel decisions, and designs the sensing, contention, aggregation and broadcasting of $\mathrm{CM}$ accordingly to achieve the aforementioned tasks.

\section{IMPLiCit COOPERATIVE SENSING}

We propose implicit cooperative sensing for energy-efficient CRSNs. The proposed implicit cooperative sensing decreases the energy consumption of channel sensing and reduces the decision making time while keeping the PNs sufficiently protected from the transmissions of the CRSN. Our implicit cooperative sensing approach is based on three main ideas: randomized channel sensing, implicit cooperation, and simplified aggregation.

\section{A. Randomized Channel Sensing}

The primary networks' MD and FA probabilities do not improve after a certain number of cooperating SUs for a given channel. However, the energy consumption and decision delay significantly increase with increasing the number of cooperating SUs. We propose a randomized channel sensing scheme which divides the CRSN into smaller subsets. Each node in a given subset randomly chooses a single channel with probability $1 / N$ to sense as shown in Fig. 1 (b). Such a technique is applicable to CRSNs because CRSNs have large number of nodes compared to typical CR networks. The proposed randomized sensing technique will have low energy consumption per node, low channel sensing time and limit the channel switching per node to only one.

\section{B. Implicit Cooperation}

In explicit cooperation, all the SUs send their individual decisions to the FC whether or not the FC will benefit from such decisions. The aggregation rule determines how much a decision is important. For example, a decision indicating the existence of the primary activity is an important decision in OR-based aggregation. Our proposed implicit cooperation approach only sends the useful 
decision(s) such that the energy consumption and delay are significantly reduced as shown in Fig. 2 (b). Furthermore, the number of contenting nodes in the contention period decreases, and consequently, less collisions and retransmissions will be encountered which further reduce the CRSN energy consumption. We next propose the implicit OR and implicit AND rules.

1) Implicit OR (I-OR) Rule: The OR rule decides the existence of PN activity based on at least one SU sending a decision declaring the existence of the PN. Hence, all the SUs that declare the PN absence give no useful information and should not transmit their decision in order to save their energy. We design the implicit OR rule such that only the nodes that declare the existence of PN sends their decisions to the aggregation point over the CCC using contention-based carrier-sense multiple access with collision avoidance (CSMA/CA).

2) Implicit AND (I-AND) Rule: The AND rule decides the absence of PN activity if at least one SU declares the absence of PN. All the SUs that declare PN existence give no useful information and need to be silence. Similar to I-OR rule, we design the implicit AND rule such that only the nodes which infer the absence of PN sends a packet to the aggregation point.

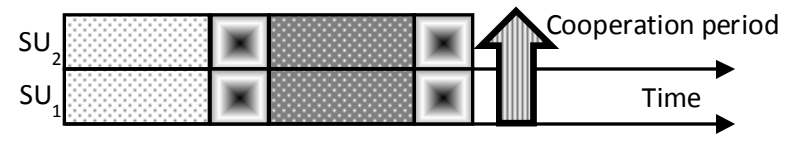

a. Typical Channel Sensing.

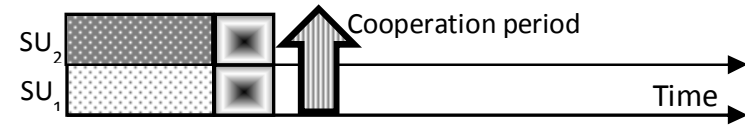

b. Randomized Channel Sensing. Sensing Ch1

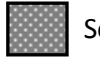
Sensing $\mathrm{Ch} 2$ Channel Switching

Fig. 1. Sensing timeline for (a) explicit and (b) implicit aggregation. All SUs sense all channels in (a) while each SU randomly senses only one channel in our proposed approach in (b).

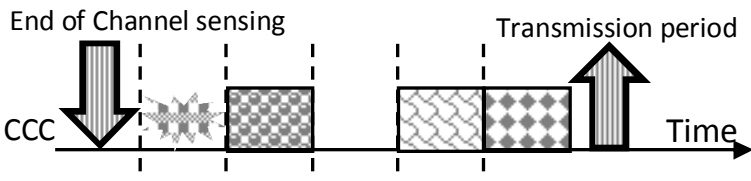

a. Typical Explicit Cooperation.
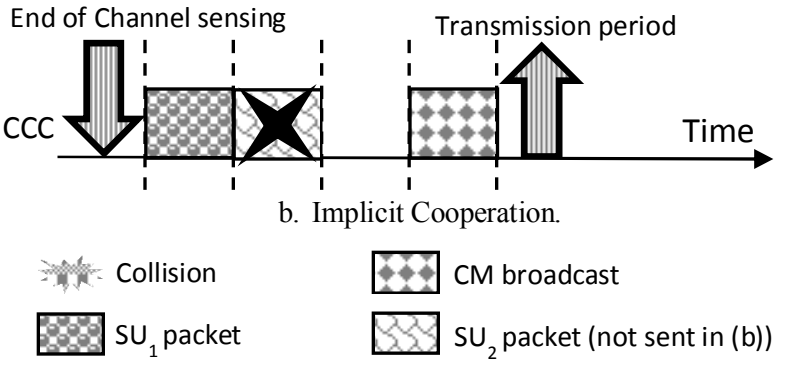

Fig. 2. Cooperation for (a) explicit and (b) implicit aggregation. In (a) all SU send their packets but in (b) a SU sends its packet only if it is contains useful information (e.g., PN is active in I-OR).

\section{Simplified Aggregation}

Implicit cooperation sends only the useful information towards the FC. Then the FC task is reduced to be only collecting and storing this information. We name this reduced FC by group master (GM) as we follow a limited centralized approach by dividing the whole CRSN network into groups. Each group elects a GM that can be any node in the network (and does not need to be with advanced capabilities) performing the implicit aggregation task temporally. When a node is in GM mode it operates only on CCC. The GM is not allowed to perform any other task until it hands over the GM task to anther node. A node self-elect itself to serve as GM as in the LEACH protocol [8]. However, the selfelection details or optimization is beyond the scope of this paper.

\section{Performance Evaluation}

\section{A. Experimental Setup}

We evaluate the performance of the proposed schemes using custom-made Matlab simulator. We consider 4 primary networks and a single CRSN composed of 8 nodes. We assume that the SU power profile follows that of a typical Atheros Wi-Fi card [9]. In such a profile, the power consumed when the node is in the receive mode is equal to the channel sensing power consumption. Since channel switching takes half the channel sensing time, the channel switching energy consumption is half the idle listening energy consumption. Transmission over the CCC is based on slotted CSMA/CA contention-based access. All channels undergo Rayleigh fading. Table I summarizes the simulation parameters.

TABLE I. Simulation Parameters

\begin{tabular}{|l|c|l|c|}
\hline \multicolumn{1}{|c|}{ Definition } & Value & \multicolumn{1}{c|}{ Definition } & value \\
\hline Number of PNs & 4 & Number of SUs & 8 \\
\hline PN TX power & $10 \mathrm{dBm}$ & Transmit Power & $127 \mathrm{~mW}$ \\
\hline Noise Floor & $-70 \mathrm{dBm}$ & Receive Power & $223.2 \mathrm{~mW}$ \\
\hline Detection Threshold & $-2 \mathrm{dBm}$ & Idle Listen Power & $219.6 \mathrm{~mW}$ \\
\hline Sensing Time & 2 slots & Sleep Power & $10.8 \mathrm{~mW}$ \\
\hline Switching Time & $1 \mathrm{slot}$ & Contention Period & 16 slots \\
\hline
\end{tabular}

\section{B. Energy Consumption}

We compare the implicit aggregation rules with the explicit aggregation rules in terms of the energy consumption per node in Watts as shown in Fig. 3. Implicit aggregation techniques consume less than half the energy consumed in explicit aggregation. The energy consumption reduction in I-OR goes from 55\% at low PN activity to $47.5 \%$ for fully backlogged PNs compared to explicit techniques due to I-OR's low packet transmission rate, collisions and retransmissions. Recall that I-OR reports the presence of PN. Consequently, it has low transmission rate at low PN activity. Meanwhile, I-AND has high transmission rate at low PN activity as it reports the absence of $\mathrm{PN}$, and hence, its energy consumption profile is opposite to I-OR with energy saving of $42.5 \%$ at low PN activity and $52.5 \%$ for fully backlogged PNs.

\section{Decision Making Delay}

Fig. 4 plots the average decision making delay per frame versus the PN activity for all aggregation techniques. Explicit aggregations show a high and fixed decision making delay since all CRSN nodes have to sense all channels are report back all their decisions. In contrary, implicit aggregations show a lower decision making delay that varies with the number of cooperating nodes since each CRSN node senses only one channel then only few 
nodes report back their decisions. I-OR gain compared to explicit techniques goes from $95 \%$ at low PN activity to $30.6 \%$ for fully backlogged PNs. Similar to the energy consumption results, I-OR and I-AND have opposite decision delay profile.

\section{Miss-Detections and False Alarms}

Fig. 5 plots the average miss-detection percentage. The AND rule has the worst performance with 16\% MD at fully backlogged PN. Recall that in AND rule if only one node decides the absence of PN (may be due to deep fade) the aggregation result is the absence of PN. I-AND rule have the next worse performance with $12 \% \mathrm{MD}$ at fully backlogged PN which is still better than AND. The OR, MAJ. and I-OR have almost the same better performance with $6 \% \mathrm{MD}$ at fully backlogged PN because these rules are more resilient to deep fading problems in some nodes.

Finally, Fig. 6 plots the average false alarm percentage. The IAND and I-OR rules show high FA at full backlogged PN with $7 \%$ and $8 \%$ respectively. The FA source is that the unreported channel is considered as busy. Random channel sensing might cause some channels to be not sensed in a given frame. The MAJ. and OR rules have better performances with 4\% FA at fully backlogged PN. The best performance is for AND rule with 3\% FA at fully backlogged PN because the probability that all nodes have a noise spark at the same time to generate a FA is small.

\section{CONCLUSION}

We have presented the I-OR and I-AND aggregation rules for energy-efficient CRSNs. The proposed schemes are based on randomized channel sensing, implicit cooperation and simplified aggregation. The proposed schemes do not only save more than half the consumed energy but also reduce the required capabilities of the aggregating node to be as simple as any ordinary node due to the low complexity of implicit aggregation. I-OR is preferred at low PN activity due to its good miss-detection performance, while I-AND is more energy saver at high PN activity above $60 \%$.

\section{REFERENCES}

[1] Zhao et al, "Energy-efficient cooperative spectrum sensing schemes for cognitive radio networks," EURASIP Journal on Wireless Communications and Networking, vol. 2013, no. 1, pp. 1-13, 2013.

[2] Y. Liu, S. Xie, Y. Zhang, R. Yu and V. C. Leung, "Energy-efficient spectrum discovery for cognitive radio green networks," Mobile Networks and Applications, vol. 17, no. 1, pp. 64-74, February 2012.

[3] S. Atapattu, C. Tellambura, H. Jiang "Conventional Energy Detector," in Energy Detection for Spectrum Sensing in Cognitive Radio, 1st ed. SpringerBriefs in Computer Science, 2014, ch. 2, pp. 11-26.

[4] H. Baradkar, and S. Akojwar, "Implementation of Energy Detection Method for Spectrum Sensing in Cognitive Radio Based Embedded Wireless Sensor Network Node," in Proc. of IEEE ICESC, 2014.

[5] I. Akyildiz, F. Brandon, R. Balakrishnan, "Co-operative spectrum sensing in Cognitive Radio networks: A survey," Physical Communications (Elesvier), vol. 4, no. 1, pp. 40-62, 2011.

[6] B. Wang and KJ R. Liu, "Advances in cognitive radio networks: A survey," Sel. Topics in Signal Processing, vol. 5, no. 1, pp. 5-23, 2011.

[7] S. Z. Y. O. Salihu, et al, "Network Layer for Cognitive Radio Sensor Networks," in Cognitive Radio Sensor Networks: Applications, Architectures, and Challenges, 1st ed. pp. 196-231, 2014.

[8] W. Heinzelman, A. Chandrakasan, and H. Balakrishnan, "EnergyEfficient Communication Protocol for Wireless Microsensor Networks," in Proc. of the $33^{\text {rd }}$ Annual Hawaii International Conference on System Sciences (HICSS), 2000

[9] X. Zhang and K. G. Shin, "E-MiLi: Energy-Minimizing Idle Listening in Wireless Networks," IEEE Transactions on Mobile Computing, vol. 11, no. 9, pp. 1441-1454, 2012.

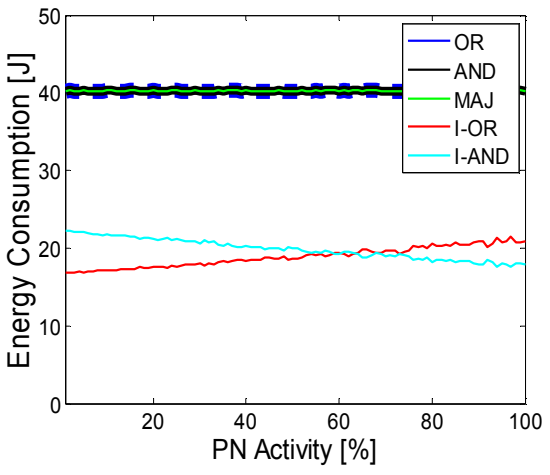

Fig. 3. Energy consumption per node.

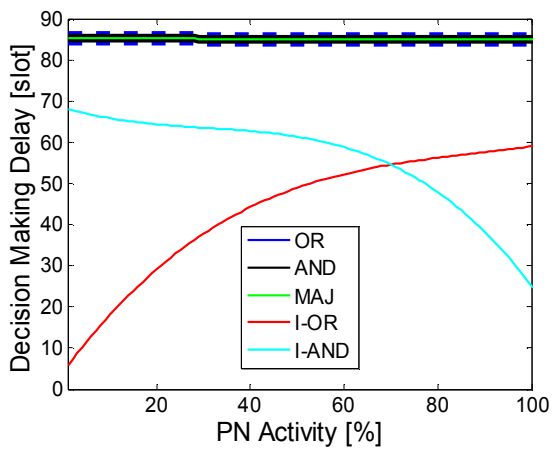

Fig. 4. Average decision making delay per sensing cycle.

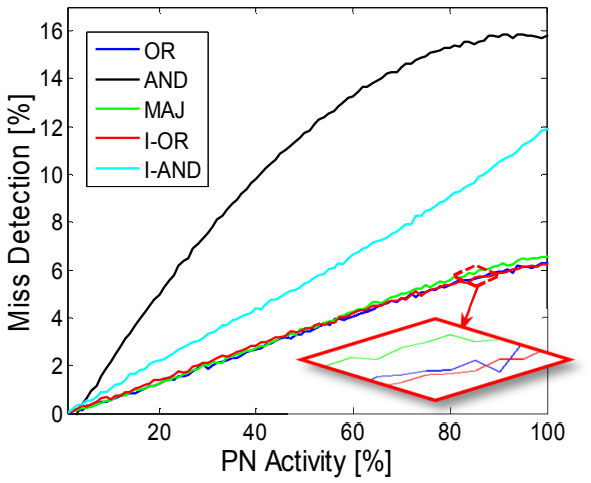

Fig. 5. Average miss-detection percentage.

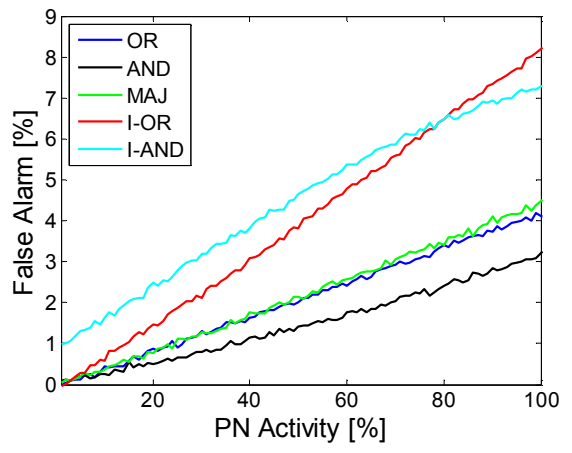

Fig. 6. Average false alarm percentage. 